\title{
Introductory editorial: December 2021
}

\author{
Michel Mandjes ${ }^{1}$
}

Received: 23 December 2021 / Revised: 24 December 2021 / Accepted: 27 December 2021 /

Published online: 22 January 2022

(c) The Author(s), under exclusive licence to Springer Science+Business Media, LLC, part of Springer Nature 2022

It is a great honor for me to succeed Professor Sergey Foss as the Editor-in-Chief of Queueing Systems: Theory and Applications (QUESTA). Sergey has had this position over the past twelve years. During his time in office, as many as 38 volumes (Nos. 64-100) have been published. He was the fourth editor-in-chief, after the journal's founder Narahari Umanath Prabhu, Richard Serfozo, and Onno Boxma.

The journal clearly is in a healthy state, very much due to Sergey's leadership as well as the continuous support of the journal's editorial board. The field has not lost any of its appeal, and in addition it has managed to connect with various surrounding disciplines. Indeed, there have been contributions on various modern queueing-related themes, some of them collected in special issues.

To ensure a smooth transition, Sergey and I will co-edit the first 2022 issues. Sergey has been of great help introducing me to the tasks the Editor-in-Chief is responsible for. In addition, regarding various more strategic issues, I have the opportunity to consult the journal's advisory board, which at the moment consists, besides my predecessors Sergey and Onno, of Venkat Anantharam, François Baccelli, Peter Glynn, and Kavita Ramanan.

I am aware that there is the continuous need to evaluate the journal's activities and policy, to make sure that its reputation and impact are kept as high as they currently are. One important issue is that I strongly feel that queueing systems should be interpreted in a broad sense. As reflected by its Aims \& Scope, the journal covers, besides queueing systems in the more conventional sense, the interfaces with various adjacent areas, such as "Markov chains and processes, stationary processes, random graphs, point processes, stochastic geometry, and related fields." In my view, the following connections will be vital in the years to come:

- One could argue that queueing systems are networks of resources that are shared by competing users. With this description in mind, there is ample space for more intensive interaction with researchers from operations management and operations research. Traditionally there has been a strong connection with engineering,

Michel Mandjes

m.r.h.mandjes@uva.nl

1 University of Amsterdam, Amsterdam, The Netherlands 
but predominantly in communications, while there are interesting ties with, for example, road network engineering as well. Methodologically, this competitionfor-scarce-resources interpretation also naturally shows the link with game theory.

- One could also interpret queues as devices that are meant to absorb fluctuations of input streams. With this description in mind, queueing theory naturally connects with, for example, the actuarial sciences and risk theory, and with the area of the analysis of extreme values. Indeed, concepts from these disciplines often have a direct queueing-theoretic counterpart.

- While traditionally queueing infrastructures were often assumed fixed, there is an increasing interest in considering their dynamic, randomly evolving, counterpartthink in this context of a road network in which sometimes accidents occur (thus temporarily blocking some links), or communication networks with link that sometimes go down. This opens up opportunities to further interact with the random graph community, with our "queueing focus" primarily on analyzing customer flows on such a random infrastructure.

- There has always been a strong connection between the queueing community and the simulation methodology community, for example, in relation to rare event estimation and variance reduction. In general, the development of efficient and accurate computational techniques has constituted an important subdiscipline within queueing theory.

- And recently, there has been an increasing interest in the connection between data and queues: the use of data to optimally control queues, the estimation of input parameters from workload observations, machine learning aspects of queueing, etc. This has led to promising interactions with the areas of artificial intelligence as well as (more traditional) statistics.

Needless to say that it is of crucial importance that QUESTA remains the preferred outlet for high-quality research on more conventional queueing theory.

I have tried to set up the editorial board so as to optimally accommodate research efforts on the interfaces mentioned above, besides the more traditional research lines in queueing theory. Indeed, the connections with, in particular, operations management, data-driven techniques, efficient simulation, network economics, and road traffic networks have been strengthened. I am also very happy about the new young members, who I hope will become the field's future research leaders. The editorial board shows balanced support from North America and Europe, with EB members from the Middle East, Australia, and Asia as well. With the support of this excellent team, I am confident that the journal's future is bright.

Publisher's Note Springer Nature remains neutral with regard to jurisdictional claims in published maps and institutional affiliations. 